\title{
NeAR Rings Admitting Certain Decomposition TheOREms
}

\author{
M. Shadab Khan \\ Department of Commerce \\ Aligarh Muslim University \\ Aligarh- 202002 (India) \\ E-mail: shadabkhan33@gmail.com
}

\begin{abstract}
In the present paper we shall investigate some decomposition theorems for near rings satisfying any one of the conditions; (1) $x y=y^{m}(x y)^{p} y^{n}$

(2) $x y=y^{m}(y x)^{p} y^{n}$, where $m=m(x, y) \geq 0, n=n(x, y) \geq 0$ and $p=p(x, y)>1$ are integers.
\end{abstract}

Keywords: Distributively Generated $(\mathrm{d}-\mathrm{g})$ near ring, D-near ring, Zero-symmetric, Zero-commutative near ring.

\section{Introduction}

The property $x^{n}=x$ has been a great favorite with many ring theorists over the last few decades since Jacobson [1] rst studied the commutativity of rings sat-isfying this condition in order to generalize the classical Wedderburn Theorem [2]. Further, Searcoid and MacHale [3] studied commutativity of rings satisfying the condition $(x y)^{n(x, y)}=x y$. Also, Ligh and Luh [4] established that a ring R satisfying the above condition is the direct sum of a J-ring and a nil ring. Later, Bell and Ligh [5] obtained a direct sum decomposition of the rings satisfying the related properties like $(x y)=(x y)^{2} f(x, y)$ for $f(X, Y) \in Z(X, Y)$ the ring of polynomials in two noncommuting indeterminate over the ring $Z$ of integers and remarked that in the case of near rings the analogous hypothesis does not quite yield a direct sum decomposition. The authors have defined a weaker notion of orthogonal sum. We say that a near ring $R$ is an orthogonal sum of sub-near rings $A$ and $B$ denoted by $R=A \oplus B$ if $A B=B A=\{0\}$ and each element of $R$ has a unique representation in the form $a+b$ with $a \in A$ and $b \in B$. The present paper is the continue study to investigate the structure of certain near rings satisfying the following conditions:

$\left(\mathrm{P}_{1}\right)$ For every $x, y \in R$ there exists integers $m=m(x, y) \geq 0, n=n(x, y) \geq 0, p=n(x, y)>1$ such that $x y=y^{m}(x y)^{p} y^{n}$.

$\left(\mathrm{P}_{2}\right)$ For every $x, y \in R$ there exists integers $m=m(x, y) \geq 0, n=n(x, y) \geq 0, p=n(x, y)>1$ such that $x y=y^{m}(y x)^{p} y^{n}$.

\section{Preliminaries}

Throughout, $\mathrm{R}$ is a left near ring with multiplicative center $\mathrm{Z}$. We shall denote by $\mathrm{N}$ the set of nilpotent elements and by $\mathrm{P}$ the set of potent elements of $\mathrm{R}$ that is $\left\{x \in R / x^{n(x)}=x\right.$, for some positive integer $\mathrm{n}(\mathrm{x})>$ $1\}$. The set of commutators is denoted by $C$. An element $x \in R$ is said to be distributive if $(y+z) x=y x+z x$ for all $y, z \in R$. A near ring $R$ is said to be distributively generated $(d-g)$ if it contains a multiplicative subsemigroup of distributive elements which generates the additive group $(R,+)$. A near ring $R$ is called periodic if for every $x \in R$ there exist distinct positive integers $m=m(x), n=n(x)$ such that $x^{m}=x^{n}$.

An ideal of a near ring $R$ is defined to be a normal subgroup I of $\left(R_{r}+\right)$ such that

(1) $\mathrm{RI} \subseteq \mathrm{I}$;

(2) $(x+i) y-x y \in I$ for all $x, y \in R$ and $i \in I$.

A near ring $R$ is called zero- commutative if $x y=0$ implies $y x=0$ for all $x, y \in R$ and if for all $x \in R, 0 x=0$ then, $R$ is called zero-symmetric (recall that left distributivity in $R$ yields $x 0=0$ ).

\section{Main Results}


Lemma 3.1. Let $R$ be a zero-symmetric near ring satisfying the following properties:

(1) For each $x \in R$, there exists a positive integer $\mathrm{n}(\mathrm{x})>1$ such that $\mathrm{x}^{\mathrm{n}}=\mathrm{x}$.

(2) Every non-trivial homomorphic image of $R$ contains a non-zero central idempotent. Then $(R,+)$ is commutative.

Lemma 3.2. Let $R$ be a zero-symmetric near ring having no non-zero nilpotent ele-ments. Then every distributive idempotent is multiplicatively central.

Lemma 3.3. Let $R$ be a zero-commutative periodic near ring, then $R=P+N$.

Lemma 3.4. Let $R$ be a near ring in which idempotent elements are multiplicatively central. If e and $f$ are any idempotent elements, then there exists an idempotent $\mathrm{g}$ such that $\mathrm{ge}=\mathrm{e}$ and $\mathrm{gf}=\mathrm{f}$.

Lemma 3.5. If $R$ is a zero-commutative near ring, then $N$ is an ideal of $R$.

Lemma 3.I to 3.5 are essentially proved in references [5- 7].

Lemma 3.6. Let $R$ be a zero-symmetric near ring satisfying either of the conditions $\left(P_{1}\right)$ and $\left(P_{2}\right)$. Then $R N=$ $N R=\{0\}$

Proof. Let $R$ satisfy $\left(P_{1}\right)$. Notice that $R$ is zero-commutative. Indeed, if $x y=0$, then there exist integers $m_{1}=m$ $(y, x) \geq 0, n_{1}=n(y ; x) \geq 0$ and $p_{1}=p(y ; x)>1$, such that $y x=x^{m 1}(y x)^{p 1} x^{n 1}=0$. Replacing, $y$ by $x$ in condition $\left(P_{1}\right)$, we get

$$
x^{2}=x^{r+2 s+t ;} ; \text { for all } r+2 s+t \geq 4 .
$$

If $u \in N$ then making repeated use of (3.1), we get $u^{2}=0$. Now for any $x \in R$, by condition $\left(P_{1}\right)$, we have $x u=$ $u^{r 1}(x u)^{s 1} u^{t 1}=0$. But, the zero-commutativity 2 of $R$ implies that $u x=0$ for $u \in N$ and $x \in R$. Hence

$$
R N=N R=\{0\}
$$

The proof of the lemma runs on the similar lines if $R$ satis fies condition $\left(P_{2}\right)$.

Theorem 3.1. Let $R$ be a zero-symmetric near ring satisfying either of the conditions $\left(P_{1}\right)$ or $\left(P_{2}\right)$. Suppose further that the idempotent elements of $R$ are multiplicatively central. Then, $P$ is a sub-near ring with $(P,+)$ abelian. $\mathrm{N}$ is a sub-near ring with trivial multiplication and $\mathrm{R}=\mathrm{P} \oplus \mathrm{N}$.

Proof. In view of Lemma 3.6, the nilpotent elements of $\mathrm{R}$ annihilate $\mathrm{R}$ on both sides and hence, in particular, $\mathrm{N}^{2}$ $=\{0\}$ and $N \subseteq Z$. Replacing $y$ by $x$ in condition $\left(P_{1}\right)$, we see that $R$ is periodic. Hence, every clement $x \in R$ can be expressed in the form $x=a+b$ where $a \in P$ and $b \in N$ by Lemma 3.3. Next, we will show that $P$ is $a$ subnear ring. Let $a, b \in P$ and choose integers $p=p(a)>1$ and $q=q(b)>1$ such that $a^{p}=a$ and $b^{q}=b$. Let

$k=(p-1) q-(p-2)=(q-1) p-(q-2)$.

Then, it is clear that $a^{k}=a$ and $b^{k}=b$. Note that $e=q^{k-1}$ and $f=b^{k-1}$ are idempotents in $R$ with ea $=a$ and $f b=$ b. Using $\left(P_{1}\right)$, we get

$a b=e a f b=(a b)(e f)=(e f)^{m}(a b c f)^{p}(e f)^{n} ;$

for some positive integers $m=m(a b$, ef $) \geq 0, n=n(a b$, ef $) \geq 0$ and $p=p(a b$, ef $) \geq 1$. Thus, we find that $a b=$ $(a b)^{p}$, hence $a b \in P$. Moreover, since $R / N$ has $x^{p}=x$ property, we have an integer $j>1$, such that

$$
(a-b)^{j}=a-b-u, u \in N(R)
$$

Using Lemma 3.4, we can choose an idempotent $\mathrm{g}$ for which ge $=\mathrm{e}$ and $\mathrm{gf}=\mathrm{f}$. Therefore, $\mathrm{ga}=\mathrm{a}$ and $\mathrm{gb}=\mathrm{b}$. Now multiplying (3.3) by $g$, we have $(a-b)^{j}=a-b$, that is $a-b \in P$. Also by the application of Lemma $3.1,(P,+)$ is abelian.

Trivially, $\mathrm{P} \cap \mathrm{N}=\{0\}$. Let $\mathrm{a}+\mathrm{u}=\mathrm{b}+\mathrm{v}$, where $\mathrm{a}, \mathrm{b} \in \mathrm{P}$ and $\mathrm{u}, \mathrm{v} \in \mathrm{N}$. Then 
$a-b=v-u \in P \cap N=\{0\}$

which yields that $\mathrm{a}=\mathrm{b}$ and $\mathrm{u}=\mathrm{v}$. Hence $\mathrm{R}=\mathrm{P} \oplus \mathrm{N}$.

The proof runs on the same lines if $R$ satisfies condition $\left(P_{2}\right)$.

The following example shows that in the hypotheses of Theorem 3.1, the centrality of idempotents is not superfluous.

Example 3.1. Let $R=\{0, a, b, c\}$ with addition and multiplication tables defined as follows.

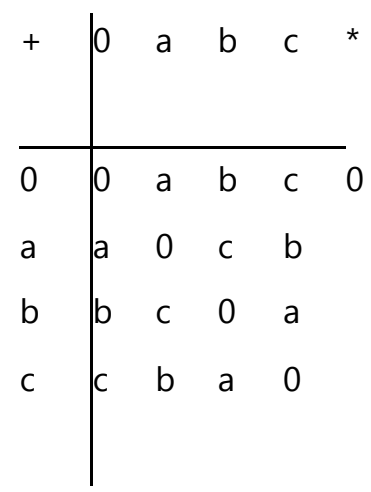

\begin{tabular}{l|lll} 
& 0a & $\mathrm{b}$ & $\mathrm{c}$ \\
& & & \\
\hline & 00 & 0 & 0 \\
$\mathrm{a}$ & $0 \mathrm{a}$ & $\mathrm{b}$ & $\mathrm{c}$ \\
$\mathrm{b}$ & $0 \mathrm{a}$ & $\mathrm{b}$ & $\mathrm{c}$ \\
$\mathrm{c}$ & $0 \mathrm{a}$ & $\mathrm{b}$ & $\mathrm{c}$
\end{tabular}

It can be easily verified that $\left(R_{1}+{ }^{*}\right)$ is a near ring satisfying both the conditions $\left(P_{1}\right)$ and $\left(P_{2}\right)$. However, the set $P=\{0, a, b\}$ is not a sub-near ring.

\section{Decomposition Theorem for D-Near Rings}

Despite the existence of such adverse examples, we need not give up the possibility of extending our Theorem 3.1 for larger classes of near rings known as D-near rings.

A near ring $R$ is called a $D$-near ring if every non-zero homomorphic image $T$ of $R$ satisfies the following conditions:

(1) T has a non-zero right distributive element;

(2) That the additive group $(T,+)$ of $T$ is abelian implies that $T$ is a ring.

All distributive and (d-g)-near rings are the examples of D-near rings. However, example 2.5 of [8] illustrates that $D$-near rings are the generalizations of $(d-g)$-near rings.

Lemma 4.1. Let $R$ be a zero-symmetric $D$-near ring. If for each $x \in R$ there exists a positive integer $n(x)>1$ such that $x^{n}=x$, then $R$ is a commutative ring.

Proof. Since $R$ is a $D$ near ring, then every non-zero homomorphic image of $R$ con-tains a non-zero distributive element, say, $a$. Choose $m=m(a)>1$ such that $a^{m}=a$, so that $a^{m-1}$ is a distributive idempotent. Also, since $R$ has no non-zero nilpotent elements, then application of Lemma 3.2 yields that $a^{m-1}$ is $a$ distributive central idempotent. Hence, by Lemma 3.1. $(R,+)$ is abelian. This together with the fact that $R$ is a $D$-near ring implies that $R$ is a ring.

Theorem 4.1. Let $R$ be a zero-symmetric $D$-near ring satisfying either of the conditions $\left(P_{1}\right)$ or $\left(P_{2}\right)$. Then $P$ is a sub-ring, $\mathrm{N}$ is a sub-near ring with trivial multiplication and $\mathrm{R}=\mathrm{P} \oplus \mathrm{N}$.

Proof. Let R satisfy condition $\left(P_{1}\right)$. In view of Lemma 3.5. R/N is a D-near ring being the homomorphic image of $\mathrm{R}$. Choose positive integers $\mathrm{m}_{1}, \mathrm{n}_{1}$, and $\mathrm{p}_{1}$ such that $\bar{x}^{m_{1}+n_{1}+p_{1}}$ for $\bar{x} \in R / N$, so that $\bar{x}\left(\bar{x}^{m_{1}+n_{1}+p_{1}-1}-\bar{x}\right)=0$. Since $R / N$ has no non-zero divisors, $\bar{x}^{m_{1}+n_{1}+p_{1}-1}=\bar{x}$, for $\bar{x} \in R / N$. Hence $R / N$ is a commutative ring by Lemma 4.1 and consequently 
Let $\mathrm{e}$ be an idempotent in $\mathrm{R}$. Then $\mathrm{e}(\mathrm{xe}-\mathrm{ex})=0$ by using (3.2), i.e. exe=ex for all $\mathrm{x}$ in R. Also by condition $\left(\mathrm{P}_{1}\right)$ there exist integers $r=r(x, e) \geq 0, s=s(x, e) \geq 0$ and $t=t(x, e)>1$ such that $x e=e^{r}(x e)^{t} e^{s}$. Multiplying by e on the left, we get exe=xe. Hence ex=xe for all $\mathrm{x}$ in R. For the rest of the proof, it is argued in the similar manner as we have done in proof of Theorem 3.1.

The proof of the theorem can be developed on the same lines if $R$ satis fies property $\left(P_{2}\right)$.

The following example due lo Clay (2.5) of reference [8] shows that one cannot get a direct sum decomposition under the hypotheses of Theorem 3.1 and Theorem 4.1.

Example 4.1. Consider non-abelian group $\left(R_{,}+\right)$isomorphic to symmetric group $S_{3}$ and de ne the multiplication in $\mathrm{R}$ as follows.

\begin{tabular}{l|lllllll}
$*$ & 0 & $a_{1}$ & $a_{2}$ & $a_{3}$ & $a_{4}$ & $a_{5}$ & \\
\hline 0 & 0 & 0 & 0 & 0 & 0 & 0 \\
$a_{1}$ & 0 & $a_{1}$ & $a_{1}$ & $a_{1}$ & & 0 & 0 \\
$a_{2}$ & 0 & $a_{1}$ & $a_{1}$ & $a_{1}$ & & 0 & 0 \\
$a_{3}$ & 0 & $a_{1}$ & $a_{1}$ & $a_{1}$ & 0 & 0 \\
$a_{4}$ & 0 & 0 & 0 & 0 & 0 & 0 \\
$a_{5}$ & 0 & 0 & 0 & 0 & 0 & 0
\end{tabular}

Then $\left(R_{,}+{ }^{*}\right)$ is a commutative near ring satisfying both conditions $\left(P_{1}\right)$ and $\left(P_{2}\right)$. However, $P=\left\{0, a_{1}\right\}$ is not an ideal of $R$.

\section{References}

[1] Jacobson N.; Structure theory of algebraic algebras of bounded degree, J. Ann. Math. 46(1945), 695 707.

[2] Wedderburn J.H.M.; A theorem on nite algebras, J. Trans. Amer. Math. Soc. 6(1905), 349-352.

[3] Searcoid M.O. and MacHale D.; J. Amer. Math. Monthly, 93(1986), 121-122.

[4] Ligh S. and Luh J.; Direct sum of J-rings and zero rings, J. Amer. Math. Monthly 96(1989), 40-41.

[5] Bell H.E. and Ligh S.; Some decomposition theorems for periodic rings and near rings, J. Math. J. Okayama Univ. 31(1989), 93-99.

[6] Bell H E.; Near ring in which each element is a power of itself, J. Bull. Austral. Math. Soc. 2(1970) 363-368.

[7] Reddy Y.F. and Murthy C.V.L.N.; Semi-symmetric ideal in near rings, Indian Journal of Pure Applied and Math. 16(1985), 17-21.

[8] Clay J. R.; The near rings on groups of low order, J. Math. Z. 104(1968), 361-37

[9] Clay J. R.; The near rings on groups of low order, J. Math. Z. 104(1968), 361-371. 